\title{
VALUATIONS AND RINGS OF QUOTIENTS
}

\author{
DAVID E. BROWN
}

\begin{abstract}
Valuations on a commutative ring, as defined by Manis, are considered in the special case where the domain of the valuation mapping is a ring of quotients of a given ring $R$. We consider relations between valuation mappings on various rings of quotients of a given ring. It is also shown that if $K$ is any von Neumann regular ring of quotients of $R$, then any pair of nonassociates of $R$ can be separated by valuations on $K$ if and only if these elements are nonassociates in the integral closure of $R$ in $K$.
\end{abstract}

1. All rings considered are commutative with a unit element. A mapping $v$ from a ring $K$ onto a totally ordered abelian group $\Gamma$ with $\infty$ adjoined is a valuation if for all $x, y \in K$ one has $v(x y)=v(x)+v(y)$ and $v(x+y) \geqq$ $\min \{v(x), v(y)\}$. We shall refer to $\Gamma \cup\{\infty\}$ as the valuation semigroup of $v$ and the set of all members of $K$ at which $v$ is not negative is called the valuation ring of $v$.

The following problem was recently considered by Max Larsen and this author [1]. Let $R$ be a ring with total quotient ring $K$, and let $\mathscr{V}$ be the set of all valuations defined on $K$ which are nonnegative on $R$. If $a$ and $b$ are any two nonassociates in $R$, can we be assured that there is a valuation $v \in \mathscr{V}$ such that $v(a) \neq v(b)$ ? In that article it was determined that $a$ and $b$ can always be separated by a valuation if $R$ is integrally closed and $K$ is (von Neumann) regular. However $K$ being regular was shown not to be a necessary condition for separation of all pairs of nonassociates of $R$ by valuations to occur.

Manis found that if $R$ is a subring of the ring $K$ and $P$ is a prime ideal of $R$, then there is a valuation ring $V$ between $R$ and $K$ which has a prime ideal $M$ such that $M \cap R=P$. No conditions were required for $K$ except that $R$ be a subring of $K$. Brown and Larsen insisted that $K$ be the total quotient ring of $R$ in order to generalize the result which can be proved about separation by valuations if $R$ is an integral domain. The results obtained in that effort have furnished a clue that favorable results may

Presented to the Society, January 26, 1973; received by the editors March 12, 1973. AMS (MOS) subject classifications (1970). Primary 13A15.

Key words and phrases. Ring of quotients, separation of nonassociates by valuations, $R$ is close to $S$. 
be possible if one allows $K$ to be any regular ring of quotients (see Lambek [4]) of $R$. In this more general setting, we will determine in $\$ 3$ exactly when, for each pair of nonassociates of $R$, there is a valuation on $K$ which is nonnegative on $R$ separating these elements. If $R$ is an integral domain, then $R$ has exactly one regular ring of quotients, namely its quotient field.

Preliminary to the result on separation, $\$ 2$ will be devoted to the following question: If $K \subset Q$ are rings with $v$ and $w$ mappings of $K$ and $Q$ into the valuation semigroups $\Gamma \cup\{\infty\}$ and $\Gamma^{\prime} \cup\{\infty\}$, respectively, such that $v$ is the restriction of $w$ to $K$, then does it follow from one being a valuation that the other is a valuation? In particular we will consider this question when $Q$ is a ring of quotients of $K$.

2. Let $v$ be a valuation on a ring $K$. We use the notation $R_{v}$ to represent the valuation ring of $v$, and $P_{v}$ represents the prime ideal of $R_{v}$ consisting of all $v$-positive elements in $K$. The valuation semigroup of $v$ is $\Gamma_{v} \cup\{\infty\}$ while $\Gamma_{v}$ is the value group of $v$. The set $v^{-1}(\{\infty\})$ is a prime ideal of both $R_{v}$ and $K$. We shall consider the valuations $v$ and $v^{\prime}$ on $K$ to be equivalent when $R_{v}=R_{v^{\prime}}, P_{v}=P_{v^{\prime}}$, and $\Gamma_{v} \cong \Gamma_{v^{\prime}}$.

Lemma 2.1. Let $K$ be a subring of the ring $Q$. If $v$ is a valuation on $K$ then there is a valuation $w$ on $Q$ such that $R_{v}=R_{w} \cap K$ and $P_{v}=P_{w} \cap K$.

Proof. Let $\Delta$ be the set of all pairs $(R, P)$ such that $R$ is a ring between $R_{v}$ and $Q$, and $P$ is a prime ideal of $R$ such that $P \cap R_{v}=P_{v}$. Since $\Delta$ is not null one can use a Zorn's lemma argument to show that there is a ring $W$ in $Q$ with a prime ideal $M$ such that $(W, M)$ is a maximal element in $\Delta$. Manis has shown that to such a pair, there corresponds a valuation $w$ on $Q$, which is unique, to within equivalence, with $W=R_{w}$ and $M=P_{w}$.

Definition. If $K \subset Q$ are rings and $w$ is a valuation on $Q$ whose restriction $v$ to $K$ is a valuation on $K$, then $w$ is an extension of $v$ to $Q$.

If the valuation pair $(W, M)$ consists of a valuation ring $W$ with associated prime $M$ for some valuation on $Q$ and $(V, P)$ is a similar pair associated with some valuation on $K$, then $(W, M)$ dominates $(V, P)$ if $V=W \cap K$ and $P=M \cap K$.

We have seen in Lemma 2.1 that if $K \subset Q$ and $(V, P)$ is a valuation pair on $K$ then there is a valuation pair $(W, M)$ on $Q$ which dominates $(V, P)$. It does not follow that if $v$ and $w$ are the valuations of $(V, P)$ and $(W, M)$, respectively, that $w$ is an extension of $v$. Since the prime ideal of elements which are infinite for a given valuation is in many cases an annihilator ideal, the next theorem is included to show a situation in which $w$ is an extension. Recall from [4] that an ideal $D$ of a ring $K$ is dense if $a D=0$ for some $a \in K$ implies that $a=0$. 
THEOREM 2.2. Let $K$ be a regular ring and $v$ a valuation on $K$ such that $v^{-1}(\{\infty\})$ is an annihilator ideal of $K$. If $Q$ is a ring of quotients of $K$, then there is a valuation $w$ on $Q$ which is an extension of $v$ and whose valuation semigroup is the same as that of $v$.

Proof. Let $\Gamma$ be the value group of $v$ and denote $v^{-1}(\{\infty\})$ by $P_{\infty}$. Since prime ideals of $K$ are maximal, there is a prime ideal $M_{\infty}$ of $Q$ with $P_{\infty}=M_{\infty} \cap K$. Define the mapping $w: Q \rightarrow \Gamma \cup\{\infty\}$ for every $q \in Q$ by $w(q)=\infty$ if $q \in M_{\infty}$ and $w(q)=v(b)-v(a)$ if $a q=b$ for some $a, b \in K$ with $a \notin M_{\infty}$.

To show that $w$ is well defined suppose that $q \notin M_{\infty}$. Since $Q$ is a ring of quotients of $K, q^{-1} K=\{r \in K \mid r q \in K\}$ is a dense ideal of $K$ and hence $q^{-1} K \not P_{\infty}$. Thus $q^{-1} K \Varangle M_{\infty}$ so there is an element $a \in q^{-1} K / M_{\infty}$ and $a q=b \notin M_{\infty}$ [2, p. 5]. Assume that also $a^{\prime}$ and $b^{\prime}$ are elements of $K$ with $a^{\prime} \notin M_{\infty}$ and $a^{\prime} q=b^{\prime}$. Then $b^{\prime} a q=b a^{\prime} q$ and $\left(b^{\prime} a-b a^{\prime}\right) q=0 \in M_{\infty}$. Since $q \notin M_{\infty}$, it follows that $b^{\prime} a-b a^{\prime} \in M_{\infty} \cap K=P_{\infty}$. Using basic properties of valuations, one can see that if $v\left(b^{\prime} a\right) \neq v\left(b a^{\prime}\right)$ then

$$
v\left(b^{\prime} a-b a^{\prime}\right)=\min \left\{v\left(b^{\prime} a\right), v\left(b a^{\prime}\right)\right\}
$$

which is finite. Hence $v\left(b^{\prime} a\right)=v\left(b a^{\prime}\right)$ and $v\left(b^{\prime}\right)-v\left(a^{\prime}\right)=v(b)-v(a)$.

The remainder of the proof is straightforward.

Again we suppose that $Q$ is a ring of quotients of $K$ and consider the following question: If one begins with a valuation $w$ on $Q$, can one say that the restriction of $w$ to $K$ is a valuation? The answer is negative as will be shown in the example following our next theorem.

THEOREM 2.3. Let $K$ be a regular subring of the ring $Q$. If $w$ is a valuation on $Q$ then the restriction of $w$ to $K$ is a valuation on $K$. Furthermore the value groups for these valuations are the same if $Q$ is a ring of quotients of $K$ and $w^{-1}(\{\infty\})$ is an annihilator ideal in $Q$.

Proof. Let $v=\left.w\right|_{K}$. It is clear that $v$ possesses the mapping properties required to be a valuation and, in fact, $v$ will be a valuation if the image $\Gamma_{v}$ of all $v$-finite elements in $K$ is a subgroup of $\Gamma_{w}$. We need only to show that $\Gamma_{v}$ is closed under subtraction.

Let $\alpha$ and $\beta$ be elements of $\Gamma_{v}$. Then there are elements $a$ and $b$ of $K$ such that $\alpha=v(a)$ and $\beta=v(b)$. Since $K$ is regular, there is an element $s \in K$ with $b^{2} s=b$. Moreover $v(b)=\beta \neq \infty$ so it follows from $2 v(b)+v(s)=v(b)$ that $v(s)=-v(b)$. Hence

$$
\alpha-\beta=v(a)+v(s)=v(a s) \in \Gamma_{v} .
$$

Now suppose that $w^{-1}(\{\infty\})$ is an annihilator ideal of $Q$. Then 
$v^{-1}(\{\infty\})=w^{-1}(\{\infty\}) \cap K$ is an annihilator ideal of $K$. Let $\gamma \in \Gamma_{w}$ and let $c$ be an element of $Q$ such that $w(c)=\gamma$. Since $Q$ is a ring of quotients of $K, c^{-1} K$ is a dense ideal of $K$. Moreover $v^{-1}(\{\infty\})$ is an annihilator ideal of $K$ so there is an element $r \in c^{-1} K$ with $v(r)=w(r) \neq \infty$. Let $d=r c \in K$. Then $\Gamma_{v}=\Gamma_{w}$ because $\gamma=w(c)=w(d)-w(r)=v(d)-v(r) \in \Gamma_{v}$.

The following example is also mentioned in [1, p. 331]. Let $F$ be the field of 2 elements. For each positive integer $k$, let $D_{k}$ be the integral domain $F[X]_{X F[X]}$, and let $P_{k}=X D_{k}$. Let $K$ be the subring of the direct product of the $D_{k}$ generated by $P=\oplus \sum P_{k}$ and 1 . Then $K$ is a total quotient ring. Let $Q$ be any regular ring of quotients of $R$. The element $a$ defined using projection maps by $\Pi_{1}(a)=X$ and $\Pi_{k}(a)=0$ if $k \neq 1$ is a member of $K$ while the quasi-inverse $a^{\prime}$ of $a$ defined by $\Pi_{1}\left(a^{\prime}\right)=1 / X$ and $\Pi_{k}\left(a^{\prime}\right)=0$ if $k \neq 1$ can be shown to be in $Q$ but not in $R$.

Define the valuation $w$ on $Q$ as follows: If $q \in Q$ then since the complete ring of quotients of $K$ is the direct product of infinitely many copies of $F(X)$, we may write $\Pi_{1}(q)=X^{n} f(X) / g(X)$ where $n$ is an integer and $f(X)$, $g(X) \in F[X]$ are relatively prime to $X$ and to each other. Thus for any $q \in Q$ with $\Pi_{1}(q) \neq 0$ we reduce $\Pi_{1}(q)$ to the above form and define $w(q)=n$. Letting $w(q)=\infty$ if $\Pi_{1}(q)=0$, we have defined a valuation $w$ on $Q$ whose value group is the set of integers. The mapping $v=\left.w\right|_{K}$ is not a valuation on $K$ since $v(a)=w(a)=1$, but even though $w\left(a^{\prime}\right)=-1$, there is no member of $K$ whose value is -1 .

It is well known (see [4]) that the complete ring of quotients of the ring $R$ is regular if and only if $R$ is semiprime. Among all of the regular rings containing the ring $R$ in a given larger ring, there is a unique minimal regular ring containing $R$ (cf. [6]). Thus Theorem 2.3 tells us that the valuations on any ring of quotients of the ring $R$ are extensions of valuations on the minimal regular ring of quotients of $R$.

3. In this section we focus our attention on a ring of quotients $K$ of $R$. The set of all valuations on $K$ which are nonnegative on $R$ is denoted by $\mathscr{V}$. If the ring $R$ has a nonzero nilpotent element $x$, then $x$ and $x^{2}$ are nonassociates which are infinite valued for all valuations in $\mathscr{V}$. Since this section is concerned with the problem of determining when a pair of nonassociates in $R$ have different values for all valuations in $\mathscr{V}$, it is assumed henceforth that all rings are semiprime. With this assumption we will always be able to find a regular ring of quotients (even unique minimal and unique maximal ones). The following preliminary result is a straightforward modification of a theorem of Griffin [3].

LEMma 3.1. The integral closure $S$ of $R$ in $K$ is the intersection of the members of $\left\{R_{v} \mid v \in \mathscr{V}\right\}$ if $K$ is a regular ring of quotients of $R$. 
The expression $\mathscr{V}$ separates nonassociates in $R$ means that given any pair of nonassociates in $R$, there is some valuation in $\mathscr{V}$ for which these elements have different values. Because we are concerned with a more general setting here than in [1], we need to make a slight modification in our definition of closeness.

Definition. If $S$ is an overring of $R$, then $R$ is close to $S$ if, when $a, b \in R$ are associates in $S$, then they are associates in $R$.

This definition reduces to our former one if $S$ lies between $R$ and its total quotient ring but is a proper extension of the previous definition in the general case.

We are now ready to prove the main result.

THEOREM 3.2. Let $R$ be a ring with $K$ a regular ring of quotients of $R$. Nonassociates in $R$ are separated by $\mathscr{V}$ if and only if $R$ is close to $S$, the integral closure of $R$ in $K$.

Proof. Assume that $R$ is not close to $S$. Let $a$ and $b$ be associates in $S$ which are not associates in $R$, say $a u=b$ with $u$ a unit in $S \backslash R$. Let $v$ be a valuation from $\mathscr{V}$. Since $v$ is nonnegative on $S$ and $u$ is a unit of $S$, $v(u)=0$. Hence $v(a)=v(a)+v(u)=v(a u)=v(b)$, so $\mathscr{V}$ does not separate nonassociates of $R$.

Conversely suppose that $R$ is close to $S$ and that $a, b \in R$ with $v(a)=v(b)$ for all $v \in \mathscr{V}$. Using the method of proof from Theorem 3 of [1], one can compute a unit $u$ in $S$ with $a u=b$. Hence $a$ and $b$ are associates in $S$, and since $R$ is close to $S, a$ and $b$ are associates in $R$.

One should observe that this theorem leaves separation of nonassociates of $R$ dependent upon one's choice of $K$. The particular integral closure of $R$ considered and the set of valuations used depend on $K$. From the results of $\S 2$, one can see that choosing $K$ to be the unique minimal regular ring of quotients of $R$ will provide the largest collection of valuations to work with. On the other hand, it is conceivable that this minimal regular ring may not contain enough units to make appropriate elements be associates. The question of whether separation is dependent on $K$ remains unresolved.

\section{BIBLIOGRAPHY}

1. D. E. Brown and M. Larsen, Separation of nonassociates by valuations, Proc. Amer. Math. Soc. 31 (1972), 326-332. MR 44 \#6695.

2. N. J. Fine, L. Gillman, and J. Lambek, Rings of quotients of rings of functions, McGill Univ. Press, Montreal, Que., 1966. MR 34 \#635.

3. M. Griffin, Prufer rings with zero divisors, J. Reine Angew. Math. 239/240 (1969), 55-67. MR 41 \#188. 
4. J. Lambek, Lectures on rings and modules, Blaisdell, Waltham, Mass., 1966. MR 34 \#5857.

5. M. E. Manis, Valuations on a commutative ring, Proc. Amer. Math. Soc. 20 (1969), 193-198. MR 38 \#2134.

6. A. C. Mewborn, Some conditions on commutative semiprime rings, J. Algebra 13 (1969), 422-431. MR 43 \#1957.

Department of Mathematics, Stephens College, Columbia, Missouri 65201

Current address: Department of Mathematics, Rockford College, Rockford, Illinois 61101 